\title{
Operando Electron Microscopy of Catalysts
}

\author{
B.K. Miller and P.A. Crozier \\ School for Engineering of Matter, Transport and Energy, Arizona State University, Tempe, AZ \\ 85287-6106
}

Linking catalyst structure with activity is a primary goal of much catalysis research; despite extensive study, these structure-activity relationships are often poorly understood. Observation of a catalyst at the atomic scale using environmental transmission electron microscopy (ETEM) during a catalytic reaction is a powerful technique for correlating structures with activity; to do this well, it is essential to measure the activity of the catalyst while it is being observed. This is known as operando TEM [1]. The activity of the catalyst can be determined by measuring the gas composition using electron energy loss spectroscopy (EELS), and/or mass spectrometry [2].

Operando TEM is a complex technique because multiple environmental parameters must be controlled, and several distinct data types collected and analyzed simultaneously. To explore the activity of the catalyst, the temperature, gas pressure, and gas composition must all be controlled. Additionally, it is essential that the effect of the electron beam on the sample be minimized, so beam dose and dose rate must also be carefully limited. Due to the dynamic nature of the experiment, all data must be recorded over time, adding a dimension to each type of data acquired. Images acquired periodically over time, or video frames continuously acquired, yield a 3D data set. 2D data sets are generated by the acquisition of mass spectra and/or EELS spectra which must be rapidly analyzed. Finally, 1D data is produced as temperature and pressure are measured as a function of time. Each of these data types are captured at different frequencies. All these sources of data must be integrated to yield a complete description of the experiment. A graphical representation of this for a single experiment which explored the catalytic oxidation of $\mathrm{CO}$ to $\mathrm{CO}_{2}$ over a $\mathrm{SiO}_{2}$-supported $\mathrm{Ru}$ catalyst is shown in Figure 1. This complexity should be managed through the development of automated systems for collection and analysis of data.

The amount of data summarized in Figure 1 is quite large. Over 8k temperature measurements, 2.5k image frames, 1.5k EELS spectra, and 55k mass spectra, all acquired at different frequencies, and thus slightly different times, are represented. It is important to keep all of this disparate data organized and easily accessible since the large amount of data must be distilled into a much smaller amount of scientifically valuable information. Finally, images, like those shown in Figure 2, must be interpreted to identify the presence or absence of catalytically relevant structures, so that these may be correlated with the activity of the catalyst. Ideally, enough data should be acquired that a statistical description of these structures becomes possible.

References:

[1] S. Chenna, P.A. Crozier, ACS Catalysis 2, (2012), 2395-2402.

[2] B.K. Miller, P.A. Crozier, Microscopy and Microanalysis 20, (2014), 815-824.

[3] J. Aßmann, V. Narkhede, N.A. Breuer, M. Muhler, A.P. Seitsonen, M. Knapp, et al., J Physics: Condensed Matter 20, (2008).

[4] The support from National Science Foundation CBET-1134464 and the use of ETEM at John M. Cowley Center for HR Microscopy at Arizona State University is gratefully acknowledged. 


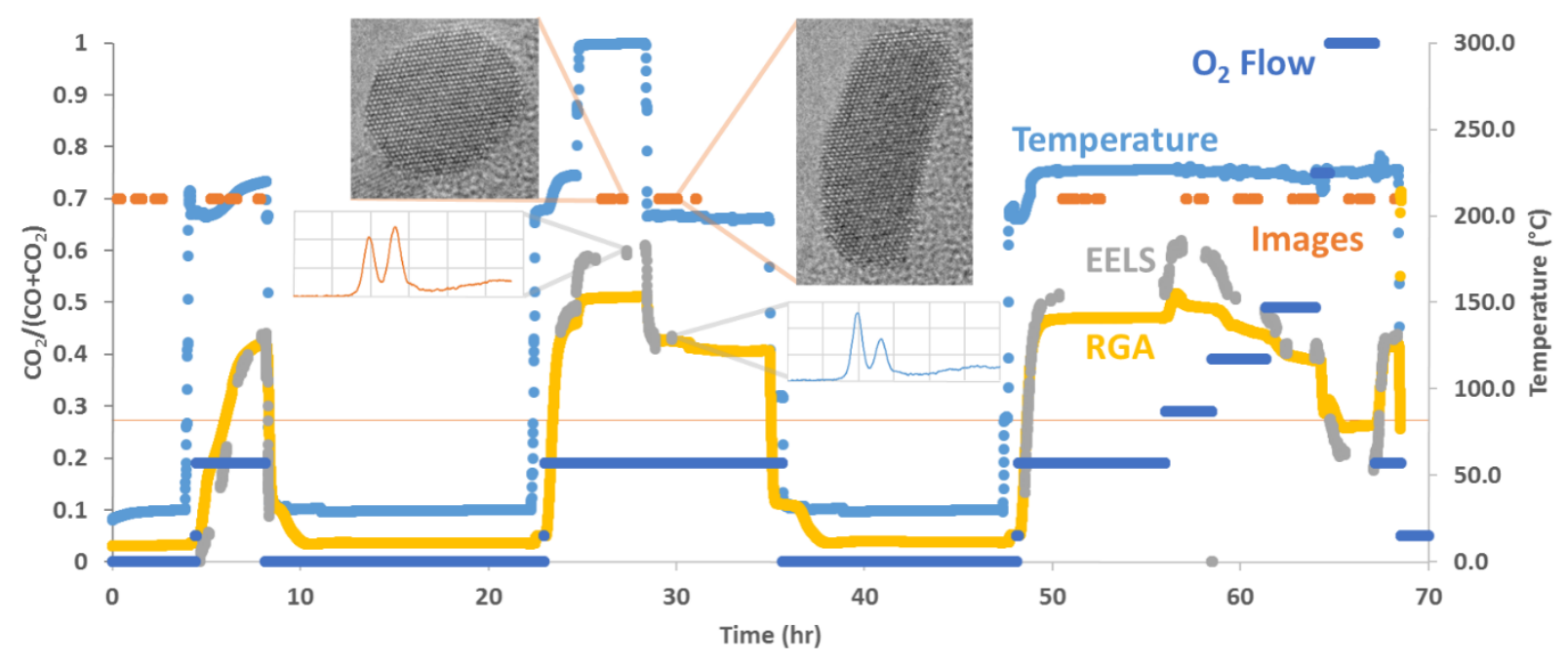

Figure 1. Plot summarizing the progression of an operando $\mathrm{CO}$ oxidation experiment over time. Temperature is plotted on the right axis, and the $\mathrm{CO}_{2}$ conversion quantified using both EELS (grey) and RGA (yellow) is on the left axis. The normalized $\mathrm{O}_{2}-\mathrm{CO}$ flow ratio is also plotted, with an orange horizontal line indicating a stoichiometric composition. The points where images of the Ru catalyst were acquired is indicated with orange markers. A few images and spectra, shown in Figure 2, are presented as thumbnails.

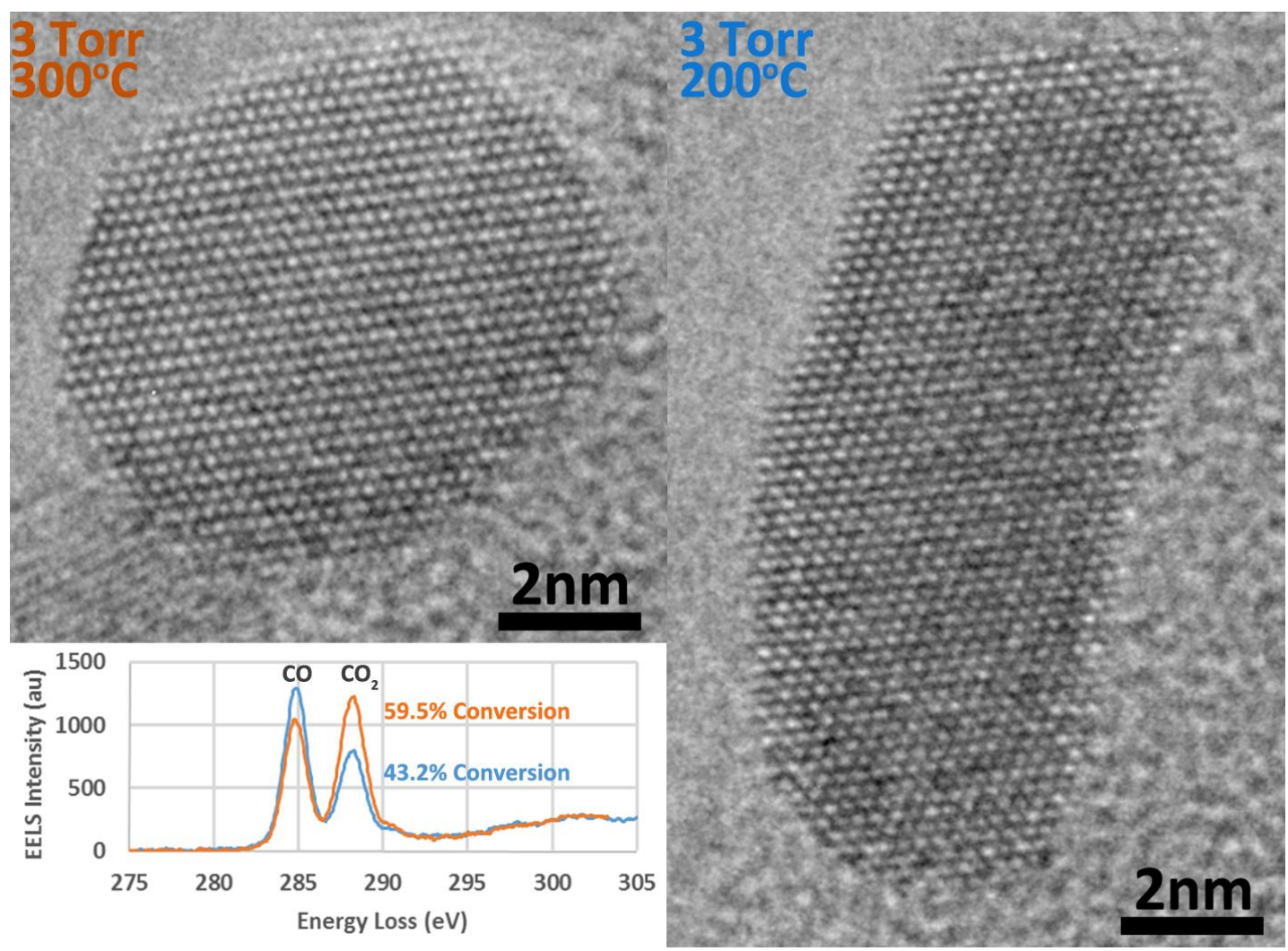

Figure 2. Two images and corresponding EELS spectra from the experiment in Figure 1. Each image is an average of 5 aligned $\left(0.5 \mathrm{~s}\right.$ exposure) frames with a dose rate of approximately $300 \mathrm{e}^{-}$ $/ \AA^{2} / \mathrm{s}\left(0.5 \mathrm{~A} / \mathrm{cm}^{2} / \mathrm{s}\right)$. Each spectrum is also an average of 5 individual spectra. 